\title{
The ES Log-normal Distribution Determined by the Einstein Median as the Scale Parameter and the Shannon Shape Parameter
}

Jiří Stávek

\begin{abstract}
The guiding principle of this contribution is the mutual interplay between the Solar gravitational field and the Maxwell-Boltzmann distribution of speeds of atoms and the observed Fraunhofer lines. We know from numerous experiments that the Newtonian gravitational constant does not depend on the atomic mass, temperature, pressure and many other particle parameters. Therefore, we should discover a universal distribution function that could be used for all atoms and their properties for a given gravitational field. We have introduced the ES lognormal distribution fully determined by the Einstein median as the scale parameter and the Shannon shape parameter $\sigma=1 / \sqrt{ } 6$. Shannon formulated this shape parameter for the log-normal distribution describing systems with the maximum entropy formation. This ES log-normal distribution function determines the most effective mutual interactions between the gravitational field and the Maxwell-Boltzmann particles. In order to make the Einstein median formula more general, we have introduced the model of the active solid angle of the source of gravity with values $1 \leq \Omega \leq 4$ steradians. We have tested this ES log-normal distribution with three datasets measured on the Solar disc and two datasets measured on the surface of the Earth using the Mössbauer effect. There were predicted some new properties of those datasets. This model might stimulate and promote new initiatives to collect new better datasets for the Solar disc and the Mössbauer effect.
\end{abstract}

Keywords - ES log-normal distribution, Einstein median, Mössbauer effect data, Shannon shape parameter, Solar gravitational redshift data.

\section{INTRODUCTION}

The Solar gravitational redshift (GRS $\odot$ ) was discovered by Lewis E. Jewell in 1896 [1], [2]. The Solar limb effect was discovered by J. Halm in 1907 [3]. The very intensive experimental and theoretical research was given to this topic by many scholars, the leading interpretation is based on the general theory of relativity of Albert Einstein predicting the solar gravitational redshift as $z=2.11 \times 10^{-6}$ and the redshifted

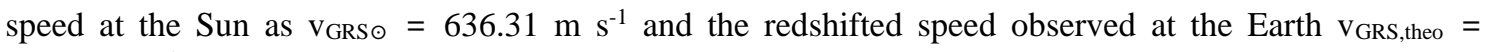
$633.10 \mathrm{~m} \mathrm{~s}^{-1}[4]$. There are known many attempts to interpret the observed spread of Solar gravitational redshifts [5]-[19].

We have discovered the key for our model in the quote of George F. FitzGerald who reacted to the discovery of the Solar gravitational redshift in 1897 as [20]: "Everybody must feel the very greatest interest in this work. It is bringing us measurably nearer a knowledge of atomic movements and interactions, the great goal of modern physical research."

We have extracted from the FitzGerald's quote following: the Solar gravitational field interplays with all Maxwell-Boltzmann particles under some hidden universal rules that can be measured by the redshifted Fraunhofer lines. Can we discover this hidden universal distribution function?

\section{BRIEF REVIEW OF OBSERVATIONS ON THE SOLAR DISC}

There were published many quantitative observations of the Solar gravitational redshifts. The dominant approach of almost all observations was to compare the observed gravitational redshifts with the prediction of Albert Einstein. Table I summarizes some published data on the redshifted speed written in the redshifts of Fraunhofer lines.

The final determination of the value of the Solar gravitational redshift depends on the selection of observed Fraunhofer lines and their position on the Solar disc. We will study the properties of our model on some historical datasets with a large number of measured lines without any selection. In our model, all

Submitted on January 06, 2022.

Published on January 28, 2022.

J. Stávek, independent researcher in Prague, Czech Republic.

(corresponding e-mail: stavek.jiri@seznam.cz) 
Fraunhofer lines contribute to the final gravitational interaction with the Solar gravitational field. The final gravitational attraction is determined by the redshifted speeds of all Maxwell-Boltzmann particles.

TABLE I: THE DETERMINATION OF THE SOLAR GRAVITATION REDSHIFT IN THE LAST 60 YeARS

\begin{tabular}{|c|c|c|c|c|}
\hline Year & $\mathrm{R} \equiv \Delta_{\text {obs }} / \Delta_{\text {theory }}$ & Number of lines & Selection of lines & Reference \\
\hline 1961 & $\mathrm{R} \approx 0.5$ (center) & Line Sr I 4607 & yes & [21] \\
\hline 1970 & $\mathrm{R}=0.61 \pm 0.06$ & Line K I 7699 & yes & [22] \\
\hline 1972 & $\mathrm{R}=1.01 \pm 0.06$ & Line K I 7699 & yes & [23] \\
\hline 1977 & $\mathrm{R}=0.97 \pm 0.02$ & Line Ti I 5713 & yes & [24] \\
\hline 1980 & $\mathrm{R}=0.76 \pm 0.24$ & 738 lines Fe I & no & [25] \\
\hline 1980 & $\mathrm{R}=0.97 \pm 0.16$ & 738 lines Fe I & Only 71 lines & [25] \\
\hline 1991 & $\mathrm{R}=0.73$ & Oxygen triplet & yes & [26] \\
\hline 1998 & $\begin{array}{l}\text { Depends on the } \\
\text { selection }\end{array}$ & 1446 Fe I lines & $\begin{array}{c}\text { Dataset of } 1446 \mathrm{Fe} \mathrm{I} \\
\text { lines }\end{array}$ & [27] \\
\hline 2012 & $\begin{array}{l}\text { Histogram of } \\
\text { redshifted speeds }\end{array}$ & 2334 lines & $\begin{array}{l}9 \text { selected lines gives } \\
\text { the theoretical value }\end{array}$ & [28] \\
\hline 2012 & $\mathrm{R}=1.10 \pm 0.19$ & $5188-5212 \AA$ & yes & [29] \\
\hline 2014 & $\mathrm{R}=0.95$ & Line K I 7699 & Observed 1976-2013 & [30] \\
\hline 2020 & $\mathrm{R}=1.009 \pm 0.009$ & $326 \mathrm{Fe} I$ lines & Selected 97 lines & [4] \\
\hline
\end{tabular}

\section{SiX HistogRAMS OF THE SOLAR GRAVITATIONAL REDSHIFTS}

We can get a good overview about the state of the situation in this field through the study of historical histograms. Fig. 1 depicts the Solar gravitational redshifted speeds based on the experimental data of Lewis E. Jewell who discovered the Solar gravitational redshift in 1896 [1], [2].

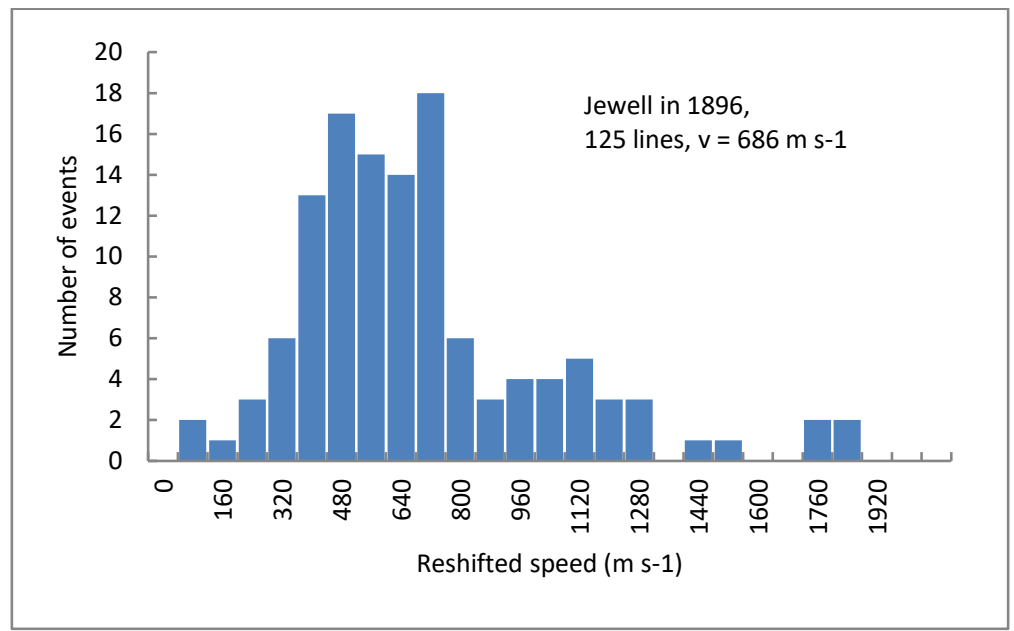

Fig. 1. Discovery of the Solar gravitational redshift by Jewell in 1896, 125 lines of different chemical elements recalculated as the redshifted speeds with the average value $v_{\text {GRS,theo }}=686 \mathrm{~m} \mathrm{~s}^{-1}$.

Fig. 2 shows the histogram of Leonhard Grebe and Albert Bachem who cooperated with Albert Einstein around the year 1920 [31]-[33].

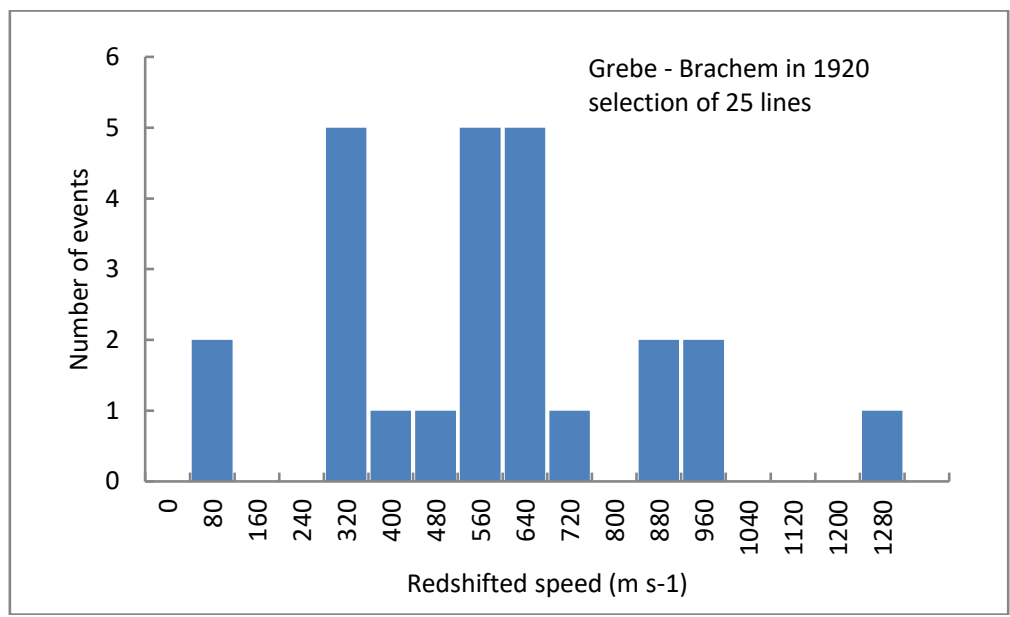

Fig. 2. Grebe - Bachem histogram in 1920 , the selection of 25 lines from 124 lines with the average value $v_{\mathrm{GRS} \text {,theo }}=540 \mathrm{~m} \mathrm{~s}^{-1}$. 
Fig. 3 brings an example of the histogram of Gonzáles Hernández from 2020 [4] where 97 Fe I lines were selected from 326 lines. These measured data were taken from light reflected from the surface of the Moon.

In the historical literature, we have studied two histograms of Charles Edward St. John [34] who was a top solar spectroscopist of that epoch working at the Mount Wilson Solar Observatory - Fig. 4 and Fig. 5 based on the data from 1928.

Fig. 5 brings the Solar gravitational redshift taken by St. John [34] close to the edge of the Solar disc to measure the Solar limb effect.

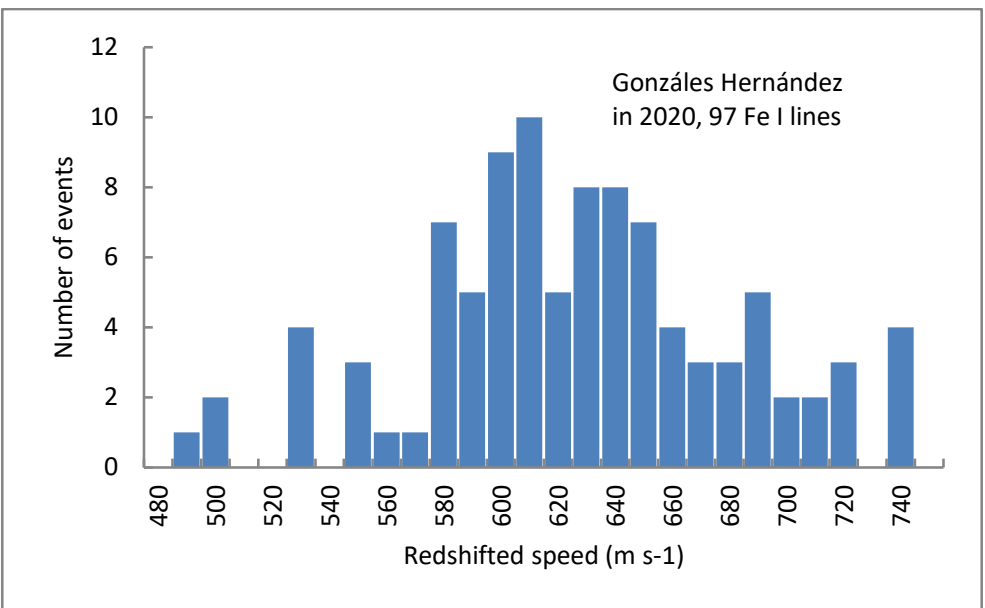

Fig. 3. Histogram of Gonzáles Hernández [4] in 2020, 97 Fe I lines were selected from 326 lines with the average redshifted speed

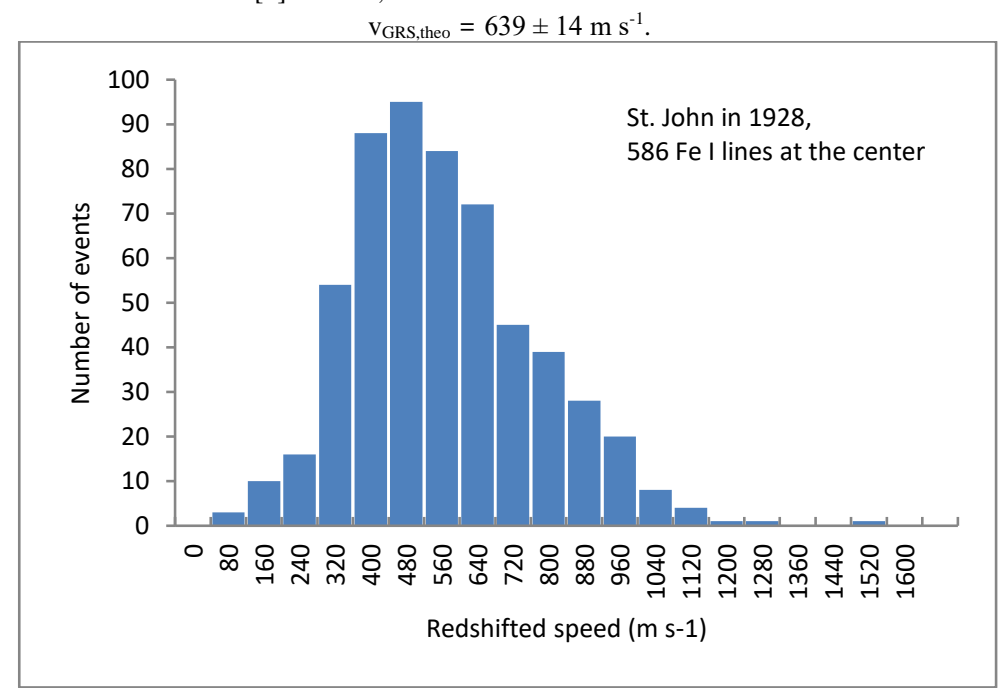

Fig. 4. St. John histogram of 586 Fe I lines [34] observed in the center of the Solar disc, the MODE redshifted speed

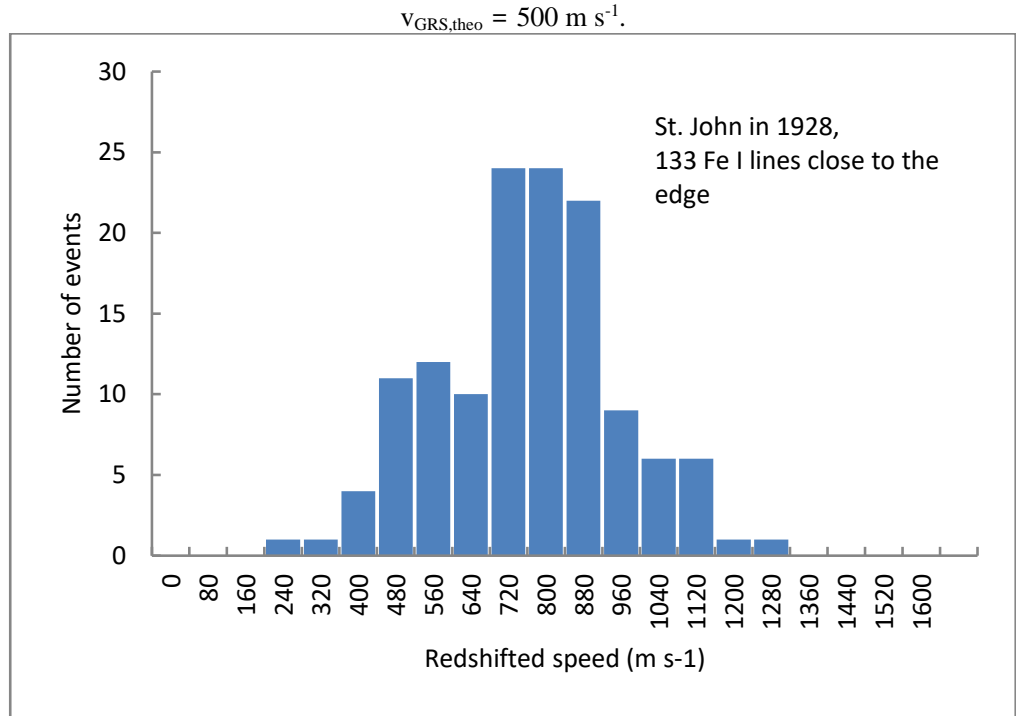

Fig. 5. St. John in 1928 [34] observed 133 Fe I lines close to the edge of the Solar disc at $0.985 \mathrm{R}_{\odot}$, the MODE redshifted speed $\mathrm{v}_{\mathrm{GRS}, \text { theo }}=800 \mathrm{~m} \mathrm{~s}^{-1}$. 
Walter S. Adams in 1910 [35] was inspired by the Solar limb effect discovered by J. Halm in 1907 [3]. Adams was also the top solar spectroscopist of that epoch working at the Mount Wilson Solar Observatory and very well equipped with instruments. Adams extracted his data closer to the edge of the Solar disc in comparison with St. John. Therefore, this dataset is more redshifted compared to two histograms of St. John.

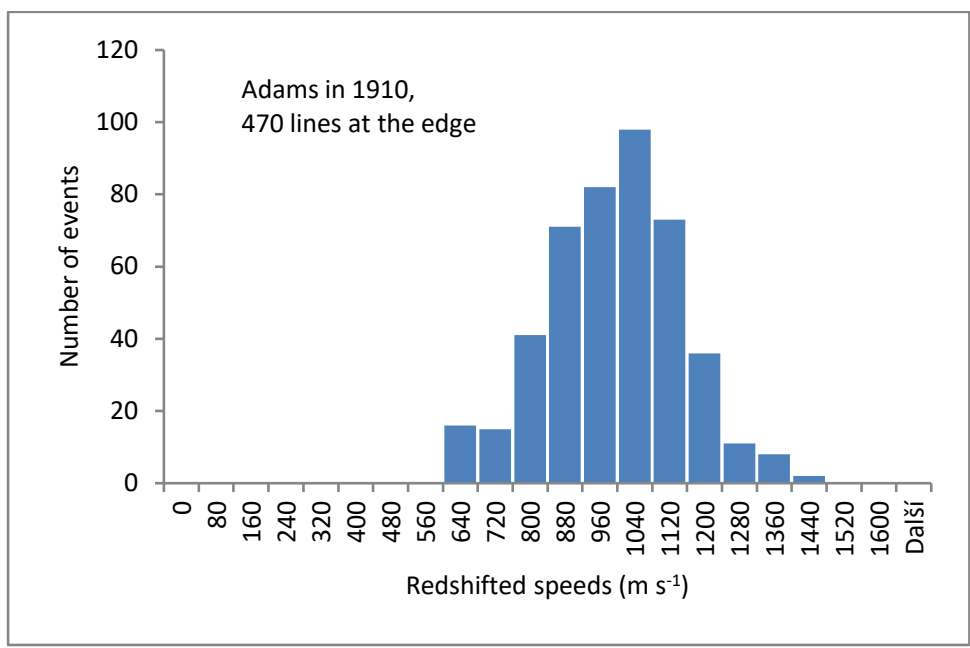

Fig. 6. Adams in 1910 observed 470 lines of different chemical elements on the edge of the Solar disc at $0.998 \mathrm{R}_{\odot}$, the MODE redshifted speed $\mathrm{v}_{\mathrm{GRS}, \text { theo }}=1040 \mathrm{~m} \mathrm{~s}^{-1}$.

\section{EINSTEIN MEdian AND THE ACTIVE SOLID ANGLE $\Omega$ OF the SuN}

Albert Einstein derived in 1915 the formula for the Solar gravitational redshift as:

$$
\frac{\lambda}{\lambda_{0}}=\frac{1}{\sqrt{1-\frac{2 G M_{\odot}}{R_{\odot} c^{2}}}} \approx 1+\frac{G M_{\odot}}{R_{\odot} c^{2}}
$$

In order to make the formula for the Einstein median more general, we propose to insert into the Einstein formula the active solid angle $\Omega$ that appears under some gravitational situations with the value different from $\Omega=2$ steradians. This more general formula can be found in the paper of Emil Wiechert in 1920 [36]:

$$
\frac{\lambda}{\lambda_{0}}=\frac{1}{\sqrt{1-\frac{\Omega G M_{\odot}}{R_{\odot} c^{2}}}} \approx 1+\frac{\Omega}{2} \frac{G M_{\odot}}{R_{\odot} c^{2}} \quad \Omega=1,2,3,4
$$

The median redshifted speed $\mathrm{v}_{\text {GRS MEDIAN }}$ for the set of the Maxwell-Boltzmann particles is given as:

$$
v_{\text {GRS MEDIA }}=\frac{\Delta \lambda}{\lambda_{0}} c=\frac{\Omega}{2} \frac{G M_{\odot}}{R_{\odot} c}
$$

The individual Maxwell-Boltzmann particles might be redshifted as:

$$
v_{\text {GRS theo }}=\omega \frac{\Delta \lambda}{\lambda_{0}} c=\omega \frac{\Omega}{2} \frac{G M_{\odot}}{R_{\odot} c} \quad 0 \leq \omega
$$

For the experimental test of this model, we have to collect redshifted speeds for all Maxwell-Boltzmann particles without any selection. The dataset should be taken on a well-defined position on the Solar disc because there might be measured a "blend" of redshifted data from different regions. The dataset of observed redshifted speeds should be as large as possible.

The active solid angle $\Omega$ can be studied in the gravitational situations given in Table II. 
TABLE II: THE ACTIVE SOLAR ANGLE $\Omega$ IN GRAVITATION SitUATIONS

\begin{tabular}{cc}
\hline \hline The active solid angle $\Omega$ & The gravitational experiment \\
$\Omega=1$ & $\begin{array}{c}\text { Transverse Mössbauer effect } \\
\text { Longitudinal Mössbauer effect } \\
\Omega=2\end{array}$ \\
$\Omega=3$ & $\begin{array}{c}\text { Fraunhofer lines in the center of the Solar disc } \\
\left(\mathrm{v}_{\mathrm{GRS}, \text { MEDIAN }}=633.10 \mathrm{~m} \mathrm{~s}^{-1}\right)\end{array}$ \\
$\Omega=4$ & $\begin{array}{c}\text { Fraunhofer lines near to the edge of the Solar disc } \\
\left(\mathrm{v}_{\mathrm{GRS}, \text { MEDIAN }}=949.65 \mathrm{~m} \mathrm{~s}^{-1}\right)\end{array}$ \\
& $\begin{array}{c}\text { Fraunhofer lines on the edge of the Solar disc } \\
\left(\mathrm{v}_{\mathrm{GRS}, \text { MEDIAN }}=1266.20 \mathrm{~m} \mathrm{~s}^{-1}\right)\end{array}$ \\
\hline \hline
\end{tabular}

\section{THE ES LOG-NORMAL DISTRIBUTION}

The Solar gravitational field is acting on all Maxwell-Boltzmann particles and causing the observed spread of redshifted speeds of individual particles towards the center of the Sun. Microscopically there should be observed a universal distribution function of those redshifted speeds but macroscopically the test mass, composed from a huge number of Maxwell-Boltzmann particles, obeys a scale parameter directing the move towards the source of the gravitational field.

For the description of the log-normal distribution we have to discover two universal parameters: the first determines the position of the log-normal distribution - the scale parameter $=$ the EINSTEIN MEDIAN. The second parameter determines the shape of the log-normal distribution curve. Claude Shannon derived for the log-normal distribution the SHANNON SHAPE PARAMETER $\sigma=1 / \sqrt{ } 6 \approx 0.4082$ based on the extremal principle of entropy. There are a lot of situations where this Shannon shape parameter determines very well the observed experimental data, e.g., [37] where is a very good introduction to this Shannon model. We assume that the Solar gravitational field interplays with all Maxwell-Boltzmann particles and the combination of microscopic redshifted speeds of individual particles results in a single macroscopic redshift speed determined by the Einstein median. The Shannon shape parameter determines the most effective cooperation between the Solar gravitational field and the individual Maxwell-Boltzmann particles of an object near the Sun. There is one more parameter "hidden" in the Einstein median - the value of the "hidden" third parameter - the ACTIVE SOLID ANGLE PARAMETER of the gravitational source $-\Omega$ should be inserted in the ES log-normal distribution in steradians. Based on the Einstein prediction scholars use only the value $\Omega=2$ sr but there are some gravitational situations where the active solid angle is $\Omega=$ $1,2,3,4$ sr.

Table III summarizes some properties of the ES log-normal distribution.

TABLE III: THE PROPERTIES OF THE ES LOG-NORMAL DISTRIBUTION

\begin{tabular}{|c|c|}
\hline NOTATION & LOG-NORMAL PROPERTIES \\
\hline Solid angle parameter $\Omega$ & $=1,2,3,4$ \\
\hline Einstein scale parameter & $\mu=\ln \left(\frac{\Omega}{2} \frac{G M_{\odot}}{R_{\odot} c}\right)$ \\
\hline Shannon shape parameter & $\sigma=\frac{1}{\sqrt{6}} \approx 0.4082$ \\
\hline PDF & $\frac{1}{x \sigma \sqrt{2 \pi}} \exp \left(-\frac{(\ln (x)-\mu)^{2}}{2 \sigma^{2}}\right)$ \\
\hline \multicolumn{2}{|c|}{ PROPERTIES FOR $\Omega=2$} \\
\hline MODE & $\exp \left(\mu-\sigma^{2}\right) \approx 535.9$ \\
\hline MEDIAN & $\exp (\mu) \approx 631.1$ \\
\hline MEAN & $\exp \left(\mu+\frac{\sigma^{2}}{2}\right) \approx 688.1$ \\
\hline SKEWNESS & $\left(\exp \left(\sigma^{2}\right)+2\right) \sqrt{\exp \left(\sigma^{2}\right)-1} \approx 1.355$ \\
\hline KURTOSIS & $\exp \left(4 \sigma^{2}\right)+2 \exp \left(3 \sigma^{2}\right)+3 \operatorname{xp}\left(2 \sigma^{2}\right)-6 \approx 3.432$ \\
\hline ENTROPY & $\mu+\frac{1}{2}+\ln (\sigma \sqrt{2 \pi}) \approx 6.974 n a t$ \\
\hline INFLECTION POINTS & $\exp \left(\mu-\frac{3}{2} \sigma^{2} \pm \frac{1}{2} \sigma \sqrt{\sigma^{2}+4}\right) \approx 325.0$ and 747.9 \\
\hline GEOMETRIC STANDARD DEVIATION & $\exp (\sigma) \approx 1.504$ \\
\hline GEOMETRIC VARIANCE & $\exp \left(\sigma^{2}\right) \approx 1.181$ \\
\hline GEOMETRIC COEFFICIENT OF VARIATION & $\exp (\sigma)-1 \approx 0.504$ \\
\hline ARITHMETIC STANDARD DEVIATION & $\exp \left(\mu+\frac{1}{2} \sigma^{2}\right) \sqrt{\exp \left(\sigma^{2}\right)-1} \approx 293.0$ \\
\hline COEFFICIENT OF VARIATION & $\sqrt{\exp \left(\sigma^{2}\right)-1} \approx 0.4258$ \\
\hline
\end{tabular}


The ES log-normal distribution determined by the Einstein median redshifted speed $\mathrm{v}_{\text {GRS MEDIAN }}=$ $631.1 \mathrm{~m} \mathrm{~s}^{-1}$ (the scale parameter), the Shannon shape parameter $\sigma=1 / \sqrt{ } 6$, and the "hidden" parameter $\Omega=$ $2 \mathrm{sr}$ is given on Fig. 7.

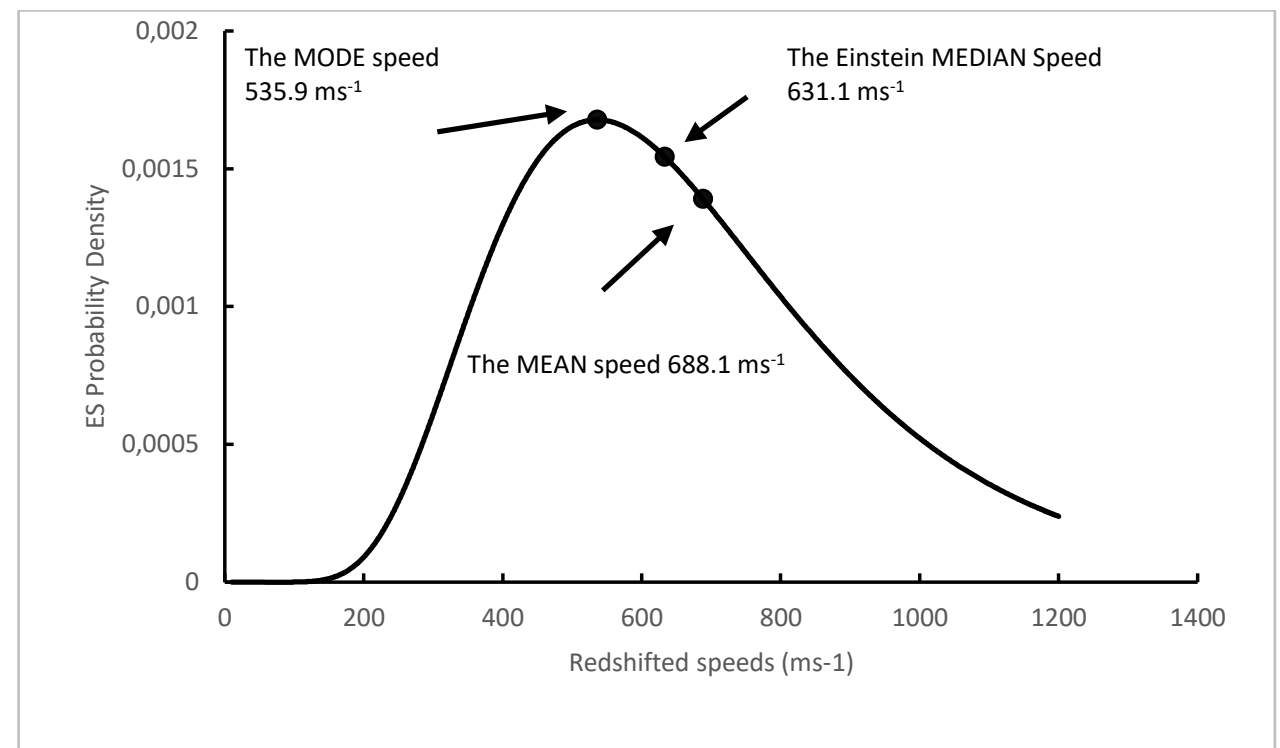

Fig. 7. The ES log-normal distribution with parameters describing the redshifted speed of the Maxwell-Boltzmann particles in the center of the Solar disc.

Under some experimental situations we cannot get experimental data for all parts of the log-normal distribution. At these situations it is possible to get the complete ES log-normal distribution from the determined values of inflection points L1 and L2 - Fig. 8.

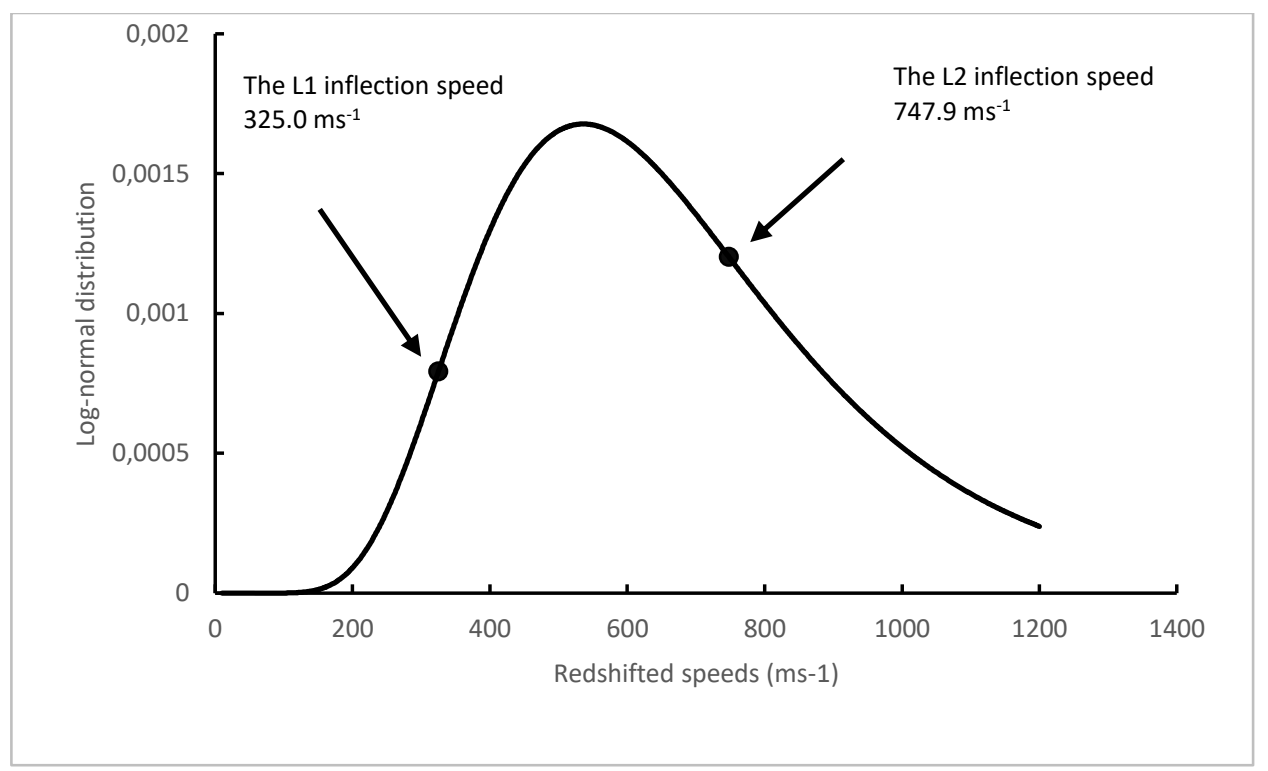

Fig. 8. The inflection points L1 and L2 could recover the complete ES log-normal distribution from an incomplete histogram.

\section{THE ES LOG-NORMAL DiSTRIBUTION IN REAL SITUATIONS}

We have tested the ES log-normal distribution on three datasets taken by top solar spectroscopists at the Mount Wilson Solar Observatory equipped with the best technology of their epoch - Walters S. Adams collected a large dataset of redshifted Fraunhofer lines on the edge of the Solar disc in 1910 and Charles Edward St. John published in 1928 two large datasets of redshifted of Fe I lines in the center of the Solar disc and close to the edge of the Solar disc.

We have evaluated from their histograms the MODE redshifted speed and recalculated as the MEDIAN redshifted speed, the shape parameter of their distribution we have determined from LN distribution of the individual redshifted speeds. The "hidden" parameter $\Omega$ was selected for that measured position on the Solar disc in order to match the observed data better than $\Omega=2$. 


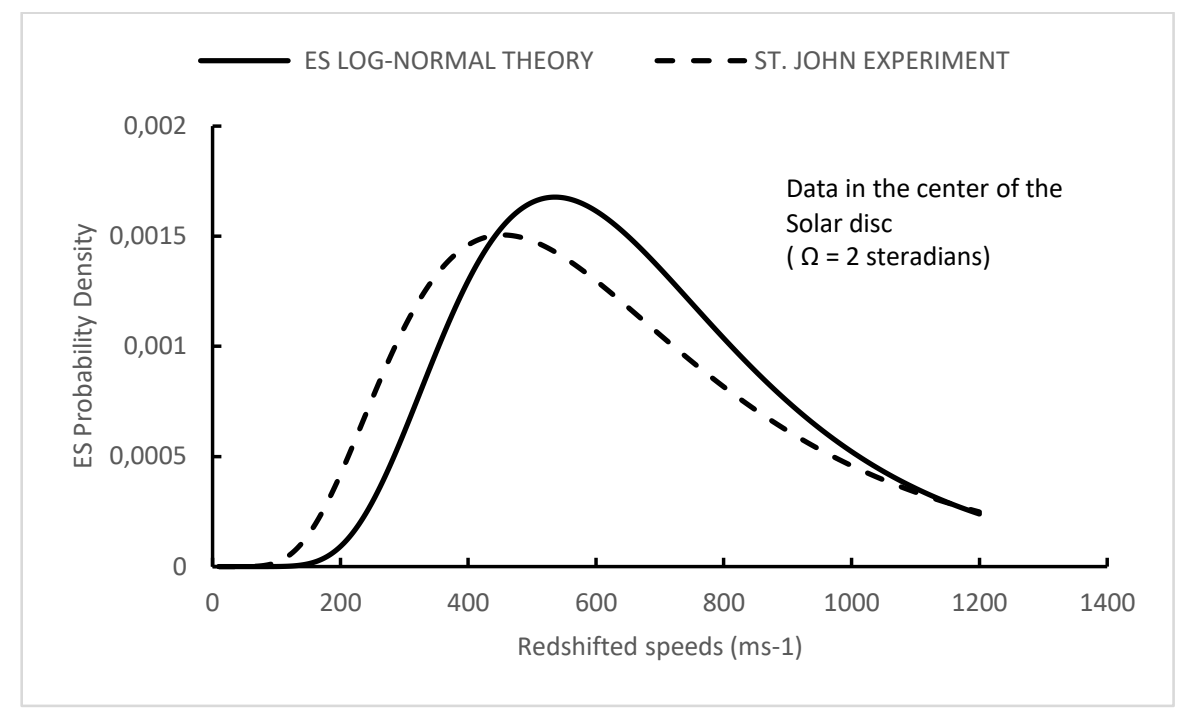

Fig. 9. ES log-normal distribution based on the dataset of St. John in 1928 [34]: 586 Fe I lines measured in the center of the Solar disc, MODE redshifted speed $500 \mathrm{~m} \mathrm{~s}^{-1}$, MEDIAN redshifted speed $590 \mathrm{~m} \mathrm{~s}^{-1}$, the shape parameter $\sigma=0.5119$, the Solar active angle $\Omega=2$.

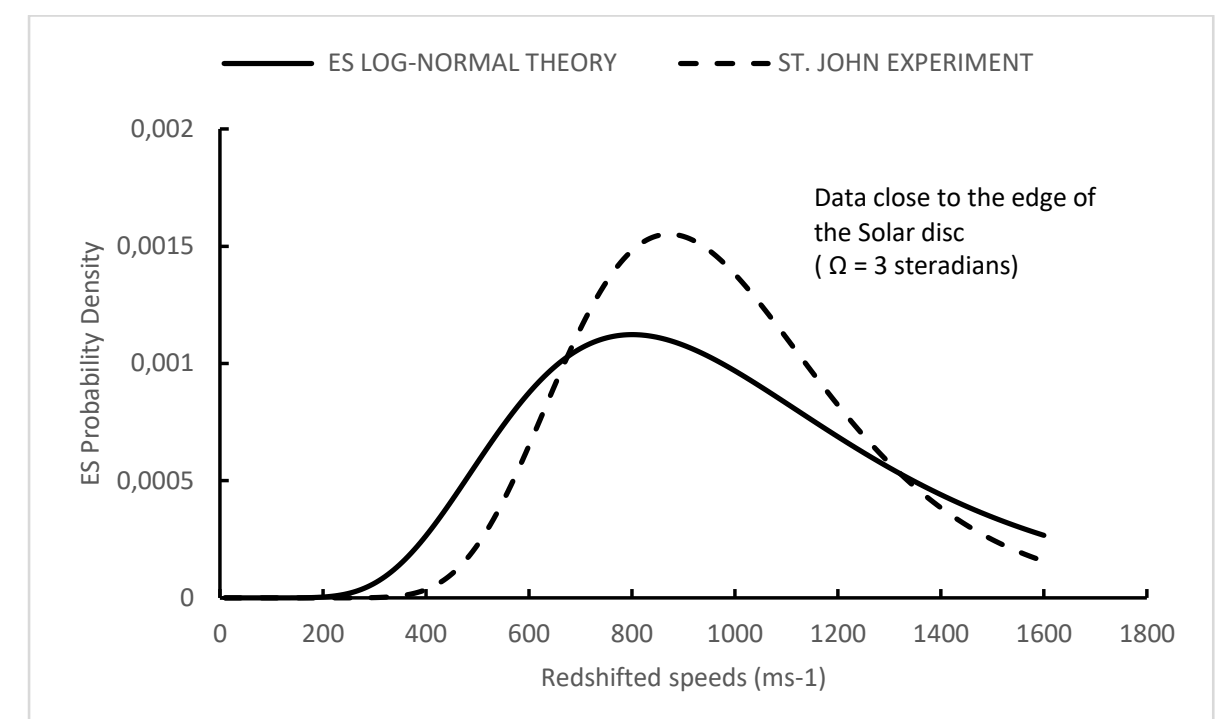

Fig. 10. ES log-normal distribution based on the dataset of St. John in 1928 [34]: 133 Fe I lines close to the edge of the Solar discs at $0.985 \mathrm{R}$, MODE redshifted speed $800 \mathrm{~m} \mathrm{~s}^{-1}$, MEDIAN redshifted speed $945 \mathrm{~m} \mathrm{~s}^{-1}$, the shape parameter $\sigma=0.2833$, the Solar active angle $\Omega=3$, (very small dataset).

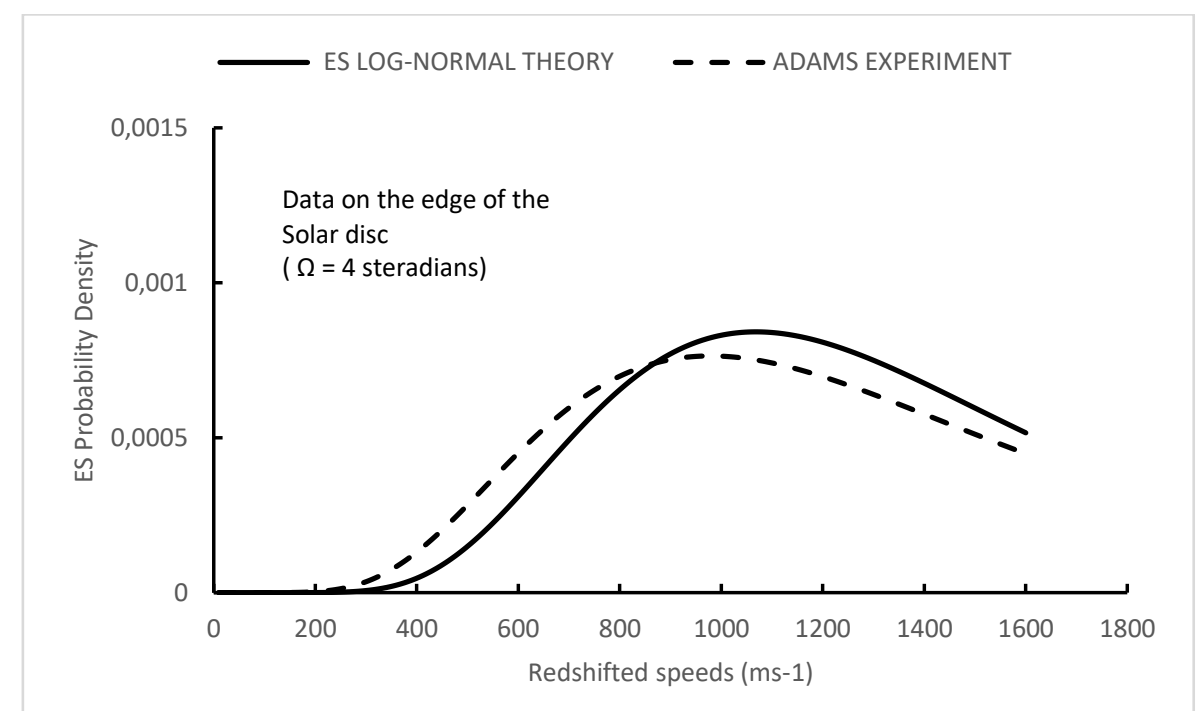

Fig. 11. ES log-normal distribution based on the dataset of Adams in 1910 [35]: 470 lines of different chemical elements on the edge of the Solar discs at $0.998 \mathrm{R}$, MODE redshifted speed $1040 \mathrm{~m} \mathrm{~s}^{-1}$, MEDIAN redshifted speed $1228 \mathrm{~m} \mathrm{~s}^{-1}$, the shape parameter $\sigma=$ 0.4764 , the Solar active angle $\Omega=4$. 
In order to better evaluate this model, it will be necessary to extract from the existing datasets larger sets of the redshifted Fraunhofer lines but with a very well-defined position on the Solar disc in order to avoid the "mixture" of redshifted Fraunhofer lines formed with different values of the active solid angle $\Omega$. On the other side, it will be necessary to add to these distribution analyses Fraunhofer lines from ultraviolet and infrared regions of spectra.

\section{ThE ES LOG-NORMAL DisTRIBUTION AND THE MÖSSBAUER EFFECT}

The measurement of the gravitational redshift using the Mössbauer effect brought a new impulse to this field [15]. We can express the observed gravitational redshift on the dependence of the height $h$ between the source and the absorber as:

$$
z=\frac{\Delta \lambda}{\lambda_{1}}=\frac{g h}{c^{2}}=h \cdot 1.0896 \cdot 10^{-16}
$$

or as the gravitationally redshifted speed $\mathrm{v}_{\text {GRS MEDIAN }}$ in $\mathrm{nm} \mathrm{s}^{-1} \mathrm{~m}^{-1}$ :

$$
v_{\text {GRS MEDIA }}=z c=\frac{g h}{c}=h \cdot 32.69\left[n \mathrm{~m} \mathrm{~s}^{-1} \mathrm{~m}^{-1}\right]
$$

Two teams of researchers significantly contributed to this topic - one team is very famous, however, the second one is almost forgotten. We can rediscover a very important information in the forgotten paper of T.E. Cranshaw and J.P Schiffer [38]. They placed the source to the top position and the absorber to the bottom and found the value $0.859 \pm 0.085$ of the Einstein prediction. We can express this experimental value as:

$$
v_{G R S \text { MODE }}=v_{\text {GRS MEDIAN }} \cdot \exp \left(\sigma^{-2}\right)=0.8465 v_{\text {GRS MEDIAN }}
$$

This interesting asymmetry in the data might reveal some deeper knowledge about the gravitational redshifts. This situation is depicted in Fig. 12.

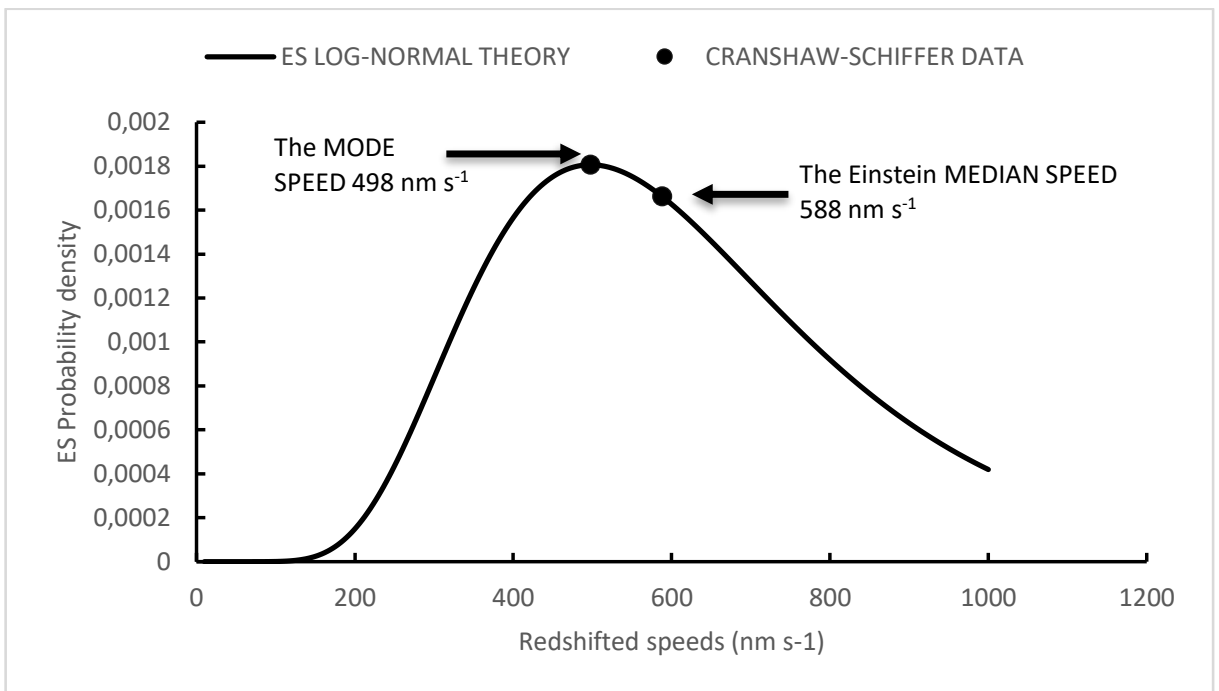

Fig. 12. Cranshaw - Schiffer observation of the gravitational redshift with the source above the absorber [38]

Therefore, we studied in details the works of Glen A. Rebka, Robert V. Pound and Joseph L. Snider in order to find similar asymmetry in their data. We have found such asymmetry in their Table I in 1960 in data before the temperature corrections [39] and in their paper [40] on page B 802. The gravitational redshift depends on the position of the source and the absorber - Fig. 13. 


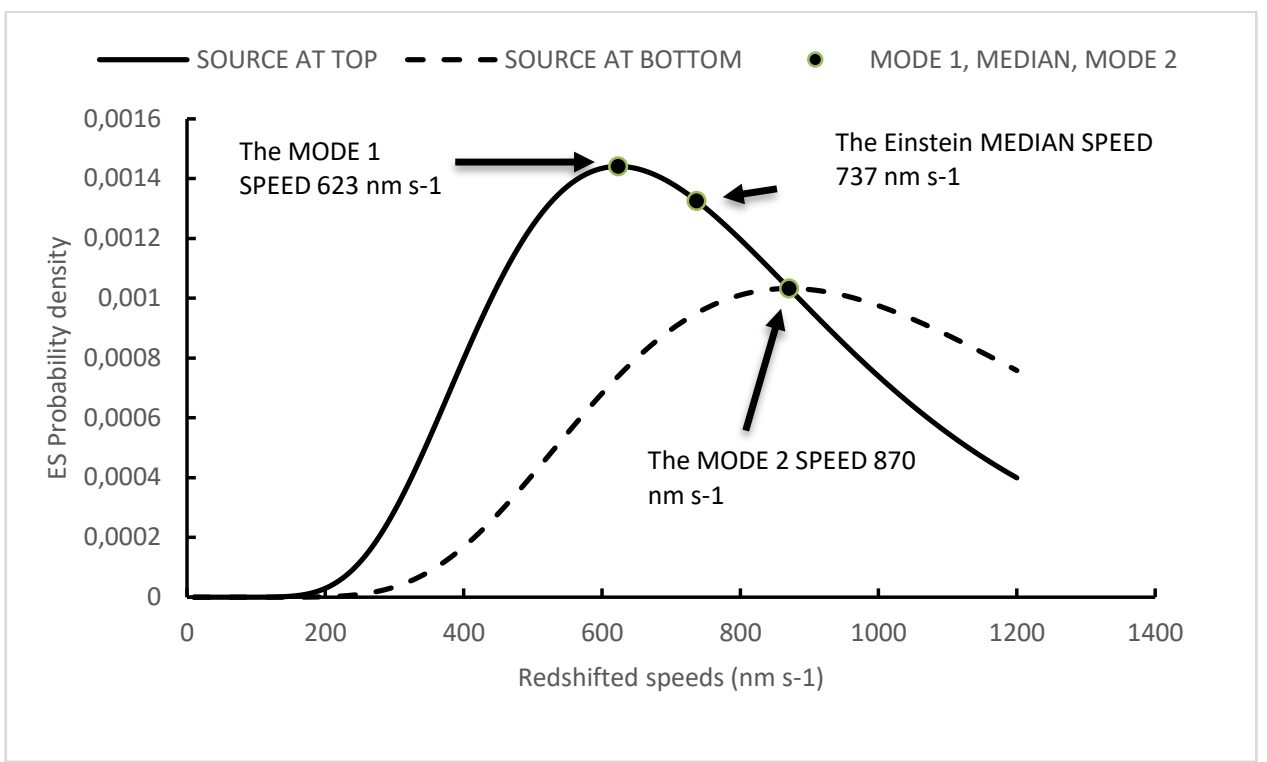

Fig. 13. Pound, Rebka, Snider data [39], [40] based on the position of the source and the absorber.

We can write a formula for two-way inspection of the gravitational redshift:

$$
v_{\text {GRS TWO-WAY }}=\sqrt{v_{\text {GRS MEDIAN }} \cdot \exp \left(\sigma^{-2}\right) v_{\text {GRS MEDIAN }} \cdot \exp \left(\sigma^{2}\right)}=v_{\text {GRS MEDIAN }}
$$

This is the reason why we will get in two-way observations of the gravitational redshift of the Einstein median with very high accuracy, e.g. [41]-[46].

\section{The Newtonian Gravitational Constant as The Macroscopic Result of Microscopic EVENTS}

There is one interesting conclusion of this contribution: we might newly view the Newtonian gravitational constant [47]-[59] as the macroscopic picture of microscopic events. For the active solid angle $\Omega=2$, we can use the Einstein median as a representative quantity for all redshifted speeds in the gravitational field of the Sun:

$$
\begin{gathered}
v_{\text {GRS MEDIAN } \odot}=\frac{\Delta \lambda}{\lambda_{0}} c=\frac{G M_{\odot}}{R_{\odot} c} \approx 636.31 \mathrm{~ms}^{-1} \\
G=\frac{v_{\text {GRS MEDIAN }} \cdot c \cdot R_{\odot}}{M_{\odot}}=G
\end{gathered}
$$

For the Earth with the redshifted speed $\mathrm{v}_{\mathrm{GRS}}$ MEDIAN $\oplus$ on the surface we can write the formula as:

$$
v_{\text {GRS MEDIAN } \oplus}=\frac{\Delta \lambda}{\lambda_{0}} c=\frac{G M_{\oplus}}{R_{\oplus} c} \approx 0.2085 \mathrm{~ms}^{-1}
$$

The acceleration $g$ on the surface of the Earth may be written as:

$$
g=v_{\text {GRS MEDIAN }} \frac{c}{R_{\oplus}}=\frac{G M_{\oplus}}{R_{\oplus}^{2}} \approx 9.8 \mathrm{~ms}^{-2}
$$

The Newtonian gravitational constant $\mathrm{G}$ for the Earth might be written as:

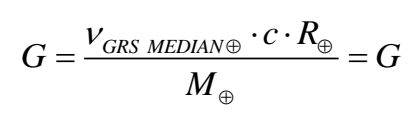


Finally, we get this expression for the Newtonian Formula for the force as:

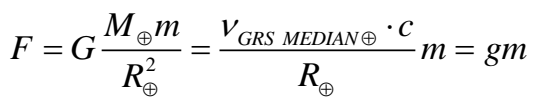

Equation 14 unites three Great Masters: Galileo, Newton and Einstein. Equation 14 describes the same gravitational event seen both from the Macrocosmos and Microcosmos.

\section{CONCLUSION}

We might open a new road leading towards the model of quantum gravity. This contribution could stimulate some new activities of the international spectroscopic community to reanalyze all existing data on the gravitational redshifts and to collect more precise and better-defined data with our existing technology. There is space enough for all participants on this Project.

\section{ACKNOWLEDGMENT}

We were supported by the contract number 0110/2020.

\section{CONFLICT OF INTEREST}

Authors declare that they do not have any conflict of interest.

\section{REFERENCES}

[1] Jewell LE. The coincidence of Solar and metallic lines. A study of the appearance of lines in the spectra of the electric arc and the Sun. The Astrophysical Journal. 1896; 3: 89-113.

[2] Hentschel K. The Discovery of the redshift of Solar Fraunhofer lines by Rowland and Jewell in Baltimore around 1890. Historical Studies in the Physical and Biological Sciences. 1993; 23: 219-277.

[3] Halm J. Über eine bisher unbekannte Verschiebung der Fraunhoferschen Linien des Sonnesspectrums. (About a hitherto unknown shift of the Fraunhofer lines of the Solar spectrum). Astronomische Nachrichten. 1907; 173: 18-287.

[4] Gonzáles Hernández JI et al. The Solar gravitational redshift from HARPS-LFC Moon spectra. Astronomy \& Astrophysics. 2020; 643: A146.

[5] Einstein A. Über das Relativitätsprinzip und die aus demselben gezogenen Folgerungen. (On the principle of relativity and the conclusions drawn from it). Jahrbuch für die Radioaktivität und Elektronik. 1907; 4: 411-462.

[6] Einstein A. Über den Einflusss der Schwerkraft auf die Ausbreitung des Lichtes (On the influence of gravitation on the propagation of light). Annalen der Physik. 1911; 340: 898-908.

[7] Einstein A. Die Grundlagen der allgemeinen Relativitätstheorie. Annalen der Physik, Series 4. 1916; 35: 115-185.

[8] Glaser LC. Die Einsteinsche Relativitätstheorie und die Rotverschiebung der Fraunhoferschen Linien. (The Einstein's theory of relativity and the redshift of Fraunhofer lines). Jahrbuch der Radioktivität und Elektronik. 1923; 20(4): 277-352.

[9] Forbes EG. The problem of the Solar redshifts. PhD Thesis. 1961; http://hdl.handle.net/10023/14628.

[10] Brault JW. The gravitational redshift in the Solar spectrum. PhD. Thesis. Princeton University. 1962.

[11] Forbes EG. A history of the solar redshift problem. Annals of Science. 1963; 17(3): 129-164.

[12] Earman J, Glymour C. The gravitational redshift as a test of general relativity: history and analysis. Studies in History and Philosophy of Science. 1980; 11: 251-278.

[13] Hentschel K. The conversion of St. John - a case of study on the interplay of theory and experiment. Science in Context. 1993; 6(1): 137-194.

[14] Hentschel K. Erwin Finlay Freundlich, Albert Einstein, and experimental tests of the general theory of relativity. Archive for History of Exact Sciences. 1994; 47(2): 143-201.

[15] Hentschel K. Measurements of gravitational redshifts between 1959 and 1971. Annals of Science. 1996; 53: $269-295$.

[16] Hentschel K. The interplay of instrumentation, experiment, and theory: patterns emerging from case studies on Solar redshift, 1890-1960. Philosophy of Science. 1997; S53-S64.

[17] Hentschel K. Zum Zusammenspiel von Instrument, Experiment und Theorie. Rotverschiebung im Sonnenspektrum und verwandte spektrale Verschiebungseffekte von 1880 bis 1960. (On the interplay of instrumentation, experiment, and theory. Redshift in the solar spectrum and related spectral shift effects from 1880 to 1960). 1998. Verlag Dr. Kovac. ISBN-10: 386064730X.

[18] Creliston J. Einstein's jury. The race to test relativity. 2006; Princeton University Press, Princeton.

[19] Treschmann KJ. Early astronomical tests of general relativity: the anomalous advance in the perihelion of Mercury and gravitational redshift. Asian Journal of Physics. 2014; 23(1\&2): 171-188.

[20] FitzGerald GF. Note on the cause for the shift of spectral lines. The Astrophysical Journal. 1897; 5: $210-211$.

[21] Blamont JE, Rodier F. Precise observation of the profile of the Frauhofer strontium resonance line. Evidence for the gravitational red shift on the Sun. Phys. Rev. Lett. 1961; 7(12): 437-439.

[22] Snider JL. Atomic-beam study of the solar $7699 \AA$ A potassium line and the Solar gravitational red-shift. Solar Physics. 1970; 12: 352-369.

[23] Snider JL. New measurement of the Solar gravitational red shift. Phys. Rev. Lett. 1972; 28(13): 853-856.

[24] Beckers JM. Material motion in sunspot umbrae. The Astrophysical Journal. 1977; 213: 900-905.

[25] LoPresto JC, Chapman RD., Sturgis EA. Solar gravitational redshift. Solar Physics. 1980; 66: 245-249.

[26] LoPresto JC, Schrader Ch, Pierce AK. Solar gravitational redshift from the infrared oxygen triplet. The Astrophysical Journal. 1991; 376: 757-760.

[27] Allende Prieto C, García lópez RJ. Fe I line shifts in the optical spectrum of the Sun. Astronomy \& Astrophysics. Suppl. Ser. 1998; 129: 41-44 
[28] Molaro P, Monai S. Solar atlas revised. Astronomy \& Astrophysics. 2012; 544: A125

[29] Takeda Y, Ueno S. Detection of gravitational redshift on the Solar disk by using iodine-cell technique. Solar Physics. 2012; 281: 551-575.

[30] Roca Cortés T, Pallé PL. The Mark-I helioseismic experiment - I. Measurements of the Solar gravitational redshift (1976-2013). Monthly Notices of the Royal Astronomical Society. 2014; 443: 1837-1848.

[31] Grebe L, Bachem A. Über die Einsteinverschiebung im Gravitationsfeld der Sonne. (On the Einstein shift in the gravitational field of the Sun). Zeitschrift für Physik. 1920; 1: 51-54.

[32] Grebe L, Bachem A. Die Einsteinsche Gravitationsverschiebung im Sonnenspektrum der Stickstoffbande $\lambda=3883$ AE. (On the Einstein gravitational shift in the Solar spectrum of the nitrogen band $\lambda=3883$ AE). Zeitschrift für Physik. $1920 ; 2$ : $415-422$.

[33] Hentschel K. Grebe/Bachems photometrische Analyse der Linienprofile und die Gravitations-Rotverschiebung: 1919 bis 1922. (Grebe/Bachem's photometric analysis of the line profile and the gravitational redshift: 1919 to 1922). Annals of Science. 1992; 49: 21-46.

[34] St. John CE. Evidence for the gravitational displacement of lines in the Solar spectrum predicted by Einstein's theory. The Astrophysical Journal. 1928; 67: 195-239.

[35] Adams WS. An investigation of the displacements of the spectrum lines at the Sun's limb. The Astrophysical Journal. 1910; 31: 30-61.

[36] Wiechert E. Die Gravitation als elektromagnetische Erscheinung. (Gravity as an electrodynamic phenomenon). Annalen der Physik. 1920; 63: 301-381, page 320.

[37] Wu Z, Li J, Bai Ch. Scaling relations of lognormal type growth process with an extremal principle of entropy. Entropy. 2017; 19(56): 1-14.

[38] Cranshaw TE, Schiffer JP. Measurement of the gravitational red shift with the Mössbauer effect. Proc. Phys. Soc. 1964; 84: 245256.

[39] Pound RV, Rebka GA. Apparent weight of photons. Physical Review Letters. 1960; 4: 337-341. Table I.

[40] Pound RV, Snider JL. Effect of gravity on gamma radiation. Physical Review. 1965; 140 (3B): B788-B804. page B802.

[41] Vessot RFC et al. Test of relativistic gravitation with a space-borne hydrogen maser. Physical Review Letters. 1980; 45: 20812084

[42] Ashby N. Relativity in the global positioning system. Living Reviews in Relativity. 2003; 6: 1.

[43] Will CM. The confrontation between general relativity and experiment. Living Reviews in Relativity. 2014;17(1): 4.

[44] Herrmann S. et al. Test of gravitational redshift with Galileo Satellites in an eccentric orbit. Physical Review Letters. 2018; 121 : 231102.

[45] Takamato M. et al. Test of general relativity by a pair of transportable optical lattice clocks. Nature Photonics. 2020; 14: 411415.

[46] Qin Ch, Tan Y, Shao ChG. Test of Einstein equivalence principle by frequency comparisons of optical clocks. Physics Letters B. 2021; 820: 136471.

[47] Sagitov MU. Current status of determinations of the gravitational constant and the mass of the Earth. Soviet Astronomy. 1970; 13:712-718.

[48] Gillies GT. The Newtonian gravitational constant: an index of measurements. Bureau International des Poids et Measureres, France. 1982.

[49] Gillies GT. The Newtonian gravitational constant: recent measurements and related studies. Rep. Prog. Phys. 1997; 60: 151225.

[50] Luo J, Hu ZK. Status of measurement of the Newtonian gravitational constant. Class. Quantum Grav. 2000; 17: 2351-2363.

[51] Milyukov VK, Luo J, Tao Ch. Mironov AP. Status of the experiments on measurement of the Newtonian gravitational constant. Gravitation and Cosmology. 2008; 14: 368-375.

[52] Milyukov V, Fan SH. The Newtonian gravitational constant: modern status of measurement and the new CODATA value. Gravitation and Cosmology. 2012; 18: 216-224.

[53] Quinn T, Speake C. The Newtonian constant of gravitation, a constant too difficult to measure? 13 contributions to a Theo Murphy Meeting Issue. Phil. Trans. R. Soc. 2014; A 372.

[54] Schlamminger S, Gundlach JH, Newman RD. Recent measurements of the gravitational constant as a function of time. Phys. Rev. D. 2015; 91: 121101R.

[55] Rothleitner C, Schlamminger S. Invited review article: measurement of the Newtonian constant of gravitation G. Rev. Sci. Instrum 2017; 88: 11110-1-111101-27.

[56] Wu J. et al. Progress in precise measurements of the gravitational constant. Annalen der Physik. 2019; 531: 1900013 (1-14).

[57] Xue C. et al. Precision measurement of the Newtonian gravitational constant. National Science Review. 2020; 12 (7): $1803-1817$.

[58] Dai DC. Variance of Newtonian constant from local gravitational acceleration measurements. Arxiv: 2103.11157v1. 2021.

[59] Quinn T. Don't stop the quest to measure Big G. Nature. 2014; 505: 455. 